\title{
Widely linear MMSE precoding and equalization techniques for SC-FDE systems
}

\author{
Bruno S Chang ${ }^{1 *}$, Carlos AF da Rocha ${ }^{2}$, Didier Le Ruyet ${ }^{3}$ and Daniel Roviras ${ }^{3}$
}

\begin{abstract}
Single-carrier systems using frequency-domain equalization (SC-FDE) systems were proposed to overcome the low robustness to carrier frequency offset (CFO) and high peak-to-average-power ratio (PAPR) inherent to regular orthogonal frequency-division multiplexing (OFDM) systems. Usually, linear minimum mean square error (MMSE) equalization is used to compensate the channel effect, since maximum likelihood (ML) detection is computationally impractical. However, if the transmitted signal comes from an improper constellation, widely linear processing can be used to take advantage of all the available second-order statistics from this transmitted signal, obtaining this way a performance gain when compared to the strictly linear case. In this paper, a SC-FDE system employing widely linear MMSE equalization is proposed in its regular and decision-feedback (DFE) versions. A SC-FDE system employing widely linear MMSE Tomlinson-Harashima precoding (THP) and equalization is also proposed. With Tomlinson-Harashima precoding, the error propagation problem observed in systems using a decision-feedback equalizer vanishes, because the feedback processing is done at the transmitter. Simulation results show that together with the error performance gain, these systems have lower sensibility to the feedback filter length in systems using decision-feedback equalizers. In Tomlinson-Harashima precoded systems, the performance gain is observed even with channel estimation/channel state information errors.
\end{abstract}

Keywords: SC-FDE; Widely linear processing; Equalization; Tomlinson-Harashima precoding

\section{Introduction}

Single-carrier systems using frequency domain equalization (SC-FDE) $[1,2]$ were proposed to reduce the computational complexity required to equalize in the timedomain single-carrier transmissions through channels with a long impulse response. When compared to orthogonal frequency-division multiplexing (OFDM) systems, they have lower peak-to-average-power ratio (PAPR) and higher robustness to carrier frequency offset (CFO) and to extreme subcarrier fading (since the decision on the symbol estimate is done in the time domain). SC-FDE-based systems were proposed for the uplink implementation in the 3GPP long-term evolution (LTE) standard.

Maximum likelihood (ML) decoding is computationally impractical for SC-FDE systems for commonly used block sizes; thus, minimum mean square error (MMSE)-based equalization is commonly used. SC-FDE systems can also

\footnotetext{
*Correspondence: bschang@utfpr.edu.br

1 Electronics Department, Federal University of Technology - Paraná, Curitiba 80230-901, Brazil
}

Full list of author information is available at the end of the article use a decision-feedback equalizer (DFE) to improve their error performance. A decision-feedback equalizer uses the previously detected symbols to reduce the postcursor intersymbol interference (ISI), which is left behind by the common MMSE equalizer. An efficient DFE structure for SC-FDE systems, using a linear frequency-domain feedforward filter and a time-domain feedback filter, was proposed in [3]. Since the feedforward filter realizes its operations in the frequency domain, its computational complexity is lower when compared to a purely timedomain DFE. In SC-FDE systems using a DFE, the effect that the error propagation can cause is limited to one symbol block, since the equalizer operates on a per-block basis.

However, this possible error propagation in the decoding process hampers the utilization of decision-feedback equalizers in systems using channel coding [4]. An alternative to avoid this problem is Tomlinson-Harashima precoding (THP) $[5,6]$, which can be seen as the implementation of the DFE receiver's feedback filter at the

\section{量 Springer}

(c) 2014 Chang et al: licensee Springer. This is an Open Access article distributed under the terms of the Creative Commons Attribution License (http://creativecommons.org/licenses/by/2.0), which permits unrestricted use, distribution, and reproduction in any medium, provided the original work is properly credited. 
transmitter. The goal of Tomlinson-Harashima precoding is to cancel the interference before transmission. Since systems using Tomlinson-Harashima precoding do not suffer from error propagation, channel coding can be applied together with precoding with a gain in error performance. As a downside, Tomlinson-Harashima precoding requires full channel state information (CSI) at the transmitter, which may be hard to obtain precisely in wireless systems. SC-FDE systems using TomlinsonHarashima precoding were proposed in $[7,8]$.

These systems normally transmit symbols from a complex quadrature amplitude modulation (QAM) constellation. QAM symbols can be described as proper, that is, they have their second-order statistics completely described by their autocovariance, which for a complex random process $\tilde{\mathbf{w}}$ with zero mean is expressed by $E\left[\tilde{\mathbf{w}} \tilde{\mathbf{w}}^{\mathrm{H}}\right]$, where $\tilde{\mathbf{w}}$ is a time-domain vector, $E\{$.$\} is the$ expectance operator, and the superscript ()$^{\mathrm{H}}$ denotes the Hermitian operator. However, if $\tilde{\mathbf{w}}$ comes from real or offset constellations (such as binary phase-shift keying (BPSK) and offset QAM (OQAM) ones), the autocovariance by itself is insufficient to describe its second-order statistics, since the pseudoautocorrelation of $\tilde{\mathbf{w}}$, given by $E\left[\tilde{\mathbf{w}} \tilde{\mathbf{w}}^{\mathrm{T}}\right]$ (where ()$^{\mathrm{T}}$ is the transpose operator) is non-zero; this type of process is called improper [9]. Widely linear (WL) processing [10-12] was proposed to take advantage of this impropriety, by processing the signal together with its conjugate version to obtain a more precise estimate. In multicarrier systems, the use of widely linear equalization to make these systems resistant to narrowband interference was proposed in [13]. A widely linear equalizer for SC-FDE systems was proposed in [14].

In this paper, we propose widely linear MMSE Tomlinson-Harashima precoders and equalizers (including a decision-feedback one) for SC-FDE systems (Section 2). An expression for the signal-to-interferenceplus-noise ratio (SINR) at the output of the receiver is provided for every proposed system (Section 3). The use of widely linear MMSE-designed equalization and precoding brings an error performance advantage with respect to strictly linear systems when improper constellations are transmitted, due to the complete use of the second-order statistics made available by these constellations. Widely linear systems are also less sensitive to the feedback filter length (in systems using decision-feedback equalizers) and channel estimation/channel state information errors (in precoded systems) when compared to systems using strictly linear processing (see Section 4).

In this paper, vectors are represented by bold lowercase letters, while bold capital letters denote matrices. Time-domain elements have a tilde appended to them. L.J represents the largest integer less than or equal to a real number. The superscripts ()$^{*},()^{\mathrm{T}}$, and ()$^{\mathrm{H}}$ denote, respectively, conjugate, transpose, and Hermitian operations.
The $i$ th element of the vector $\mathbf{v}$ is given by $v_{i}$, and the $(i, j)$ th element of the matrix $\mathbf{M}$ is given by $M_{i, j}$. The identity matrix of size $N \times N$ is denoted by $\mathbf{I}_{N}$. Finally, the expectance operator is given by $E\{$.$\} .$

\section{System model}

On the transmitter side, the block $\tilde{\mathbf{s}}=\left[\begin{array}{llll}\tilde{s}_{1} & \tilde{s}_{2} \ldots \tilde{s}_{N}\end{array}\right]^{\mathrm{T}}$ of size $N$ is composed by symbols $\tilde{s}_{i}$ belonging to an improper constellation (such as $M$-PAM or $M^{2}$-OQAM). The transmitted signal will pass through a channel with an impulse response $\tilde{\mathbf{h}}=\left[\tilde{h}_{1} \tilde{h}_{2} \ldots \tilde{h}_{L_{\tilde{\mathbf{h}}}}\right]^{\mathrm{T}}$ of size $L_{\tilde{\mathbf{h}}}$. Thus, the cyclic prefix appended to the block $\tilde{\mathbf{s}}$ before transmission must have a length $L_{\mathrm{CP}}$ of at least $L_{\tilde{\mathbf{h}}}-1$, resulting in $\tilde{\mathbf{s}}_{\mathrm{CP}}$ of length $N+L_{\mathrm{CP}}$. Complex proper uncorrelated additional white Gaussian noise (AWGN) $\tilde{\mathbf{n}}$ with zero mean and variance $\sigma_{n}^{2}$ also contaminates the transmitted signal.

Due to the cyclic prefix, the $N \times N$ channel matrix $\mathbf{H}^{\prime}$ is circulant, with its first column containing the channel impulse response $\tilde{\mathbf{h}}$ appended by $\left(N-L_{\tilde{\mathbf{h}}}\right)$ zeros. Since $\mathbf{H}^{\prime}$ is a circulant matrix, we can apply an eigendecomposition to this matrix to obtain $\mathbf{W}^{*} \mathbf{H W}$, where $\mathbf{W}$ is the normalized discrete Fourier transform (DFT) matrix of size $N \times N$ and $\mathbf{H}$ is a $N \times N$ diagonal matrix with its $(k, k)$ th entry $H_{k, k}$ corresponding to the $k$ th coefficient of the $N$-sized DFT of the channel impulse response $\tilde{\mathbf{h}}$.

The signal $\tilde{\mathbf{r}}=\left[\begin{array}{llll}\tilde{r}_{1} & \tilde{r}_{2} & \ldots & \tilde{r}_{N+L_{\mathrm{CP}}}\end{array}\right]^{\mathrm{T}}$ with length $N+L_{\mathrm{CP}}$ at the entry of the receiver has its cyclic prefix removed and passes to the frequency domain through a fast Fourier transform (FFT), so that equalization can be done in the frequency domain. This will result in the signal $\mathbf{r}$ of length $N$, expressed as

$$
\begin{aligned}
\mathbf{r} & =\mathbf{H} \mathbf{s}+\mathbf{n} \\
& =H W \tilde{s}+W \tilde{\mathbf{n}},
\end{aligned}
$$

where $\mathbf{H}$ corresponds to the channel frequency response of a specific channel realization, $\mathbf{s}=\mathbf{W} \tilde{\mathbf{s}}$ is the transmitted signal in the frequency domain, and $\mathbf{n}=\mathbf{W} \tilde{\mathbf{n}}$ is the noise in the frequency domain. Equalization is performed by filters based on the MMSE criterion. However, since the equalizer is dealing with a signal from an improper constellation (which has non-zero pseudocorrelation), it has to employ widely linear processing to use all the second-order statistics made available by the received signal. In order to do that, the original version of the received signal in the frequency domain together with its conjugate version should be processed by the equalizer.

\subsection{WL-MMSE equalizer}

The system model for a SC-FDE system employing widely linear MMSE-based equalization is presented in Figure 1. 


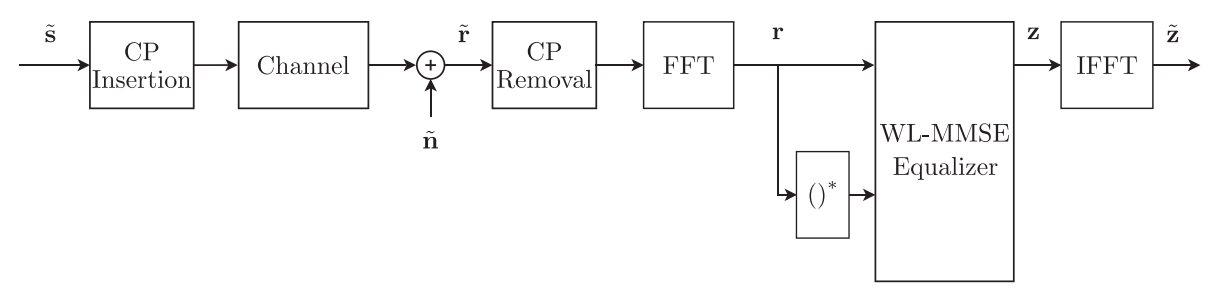

Figure 1 A SC-FDE system employing widely linear MMSE equalization.

The signal at the output of the equalizer $\mathbf{z}$, with size $N$, is given by

$$
\mathbf{z}=\mathbf{A}_{1}^{\mathrm{H}} \mathbf{r}+\mathbf{A}_{2}^{\mathrm{H}} \mathbf{r}^{*}=\mathbf{A}^{\mathrm{H}} \mathbf{t},
$$

with $\mathbf{A}^{\mathrm{H}}=\left[\mathbf{A}_{1}^{\mathrm{H}} \mathbf{A}_{2}^{\mathrm{H}}\right]$ of size $N \times 2 N$ and $\mathbf{t}=\left[\begin{array}{c}\mathbf{r} \\ \mathbf{r}^{*}\end{array}\right]$ of size $2 N$.

The cost function $\epsilon_{\mathrm{WL}}$ to derive the equalizer $\mathbf{A}$ based on the WL-MMSE criterion is

$$
\begin{aligned}
\epsilon_{\mathrm{WL}} & =E\left[\left\|\mathbf{A}^{\mathrm{H}} \mathbf{t}-\mathbf{s}\right\|^{2}\right] \\
& =\mathbf{A}^{\mathrm{H}} \mathbf{C}_{\mathbf{t t}} \mathbf{A}-\mathbf{A}^{\mathrm{H}} \mathbf{C}_{\mathbf{t s}}-\mathbf{C}_{\mathbf{s t}} \mathbf{A}+\mathbf{I}_{N} .
\end{aligned}
$$

where

$$
\begin{aligned}
& \mathbf{C}_{\mathbf{t t}}=E\left[\mathbf{t t}^{\mathrm{H}}\right] \\
& =E\left\{\left[\begin{array}{c}
\mathbf{r} \\
\mathbf{r}^{*}
\end{array}\right]\left[\begin{array}{ll}
\mathbf{r}^{\mathrm{H}} & \mathbf{r}^{\mathrm{T}}
\end{array}\right]\right\}=\left[\begin{array}{ll}
\mathbf{C}_{\mathbf{r r}} & \overline{\mathbf{C}}_{\mathbf{r r}} \\
\overline{\mathbf{C}}_{\mathbf{r r}}^{*} & \mathbf{C}_{\mathbf{r r}}^{*}
\end{array}\right] \\
& \mathbf{C}_{\mathbf{r r}}=E\left[\mathbf{r r}^{\mathrm{H}}\right]=E\left[(\mathbf{H s}+\mathbf{n})\left(\mathbf{n}^{\mathrm{H}}+\mathbf{s}^{\mathrm{H}} \mathbf{H}^{\mathrm{H}}\right)\right] \\
& =\mathbf{H} \mathbf{H}^{\mathrm{H}}+\sigma_{n}^{2} \mathbf{I}_{N} \\
& \overline{\mathbf{C}}_{\mathbf{r r}}=E\left[\mathbf{r} \mathbf{r}^{\mathrm{T}}\right]=E\left[(\mathbf{H s}+\mathbf{n})\left(\mathbf{n}^{\mathrm{T}}+\mathbf{s}^{\mathrm{T}} \mathbf{H}^{\mathrm{T}}\right)\right] \\
& =\mathbf{H U H}^{\mathrm{T}}
\end{aligned}
$$

with $\mathbf{U}$ expressed by

$$
\begin{aligned}
\mathbf{U} & =\left[\begin{array}{ccccc}
1 & 0 & 0 & \ldots & 0 \\
0 & 0 & 0 & \ldots & 1 \\
\vdots & \vdots & \vdots & \vdots & \vdots \\
0 & 0 & 1 & \ldots & 0 \\
0 & 1 & 0 & \ldots & 0
\end{array}\right], \\
\mathbf{C}_{\mathbf{t s}} & =E\left[\mathbf{t s}^{\mathrm{H}}\right]=E\left\{\left[\begin{array}{c}
\mathbf{r} \\
\mathbf{r}^{*}
\end{array}\right] \mathbf{s}^{\mathrm{H}}\right\}=E\left\{\left[\begin{array}{c}
\mathbf{r s}^{\mathrm{H}} \\
\mathbf{r}^{*} \mathbf{s}^{\mathrm{H}}
\end{array}\right]\right\} \\
& =\left[\begin{array}{c}
\mathbf{H} \\
\mathbf{H}^{*} \mathbf{U}
\end{array}\right]
\end{aligned}
$$

and

$$
\begin{aligned}
\mathbf{C}_{\mathbf{s t}} & =E\left[\mathbf{s t}^{\mathrm{H}}\right]=E\left\{\mathbf{s}^{\mathrm{H}}\left[\begin{array}{c}
\mathbf{r} \\
\mathbf{r}^{*}
\end{array}\right]\right\}=E\left\{\left[\begin{array}{c}
\mathbf{s} \mathbf{r}^{\mathrm{H}} \\
\mathbf{s}^{*} \mathbf{r}^{\mathrm{H}}
\end{array}\right]\right\} \\
& =\left[\begin{array}{c}
\mathbf{H} \\
\mathbf{U} \mathbf{H}^{*}
\end{array}\right] \\
& =\mathbf{C}_{\mathbf{t s}}
\end{aligned}
$$

with $E\left[\mathbf{n n}^{\mathrm{T}}\right]=0$ (since the noise is proper), and $\mathbf{W} \mathbf{W}^{\mathrm{H}}=$ $\mathbf{I}_{N}$. We obtain the optimal equalizer $\mathbf{A}$ by differentiating $\epsilon_{\mathrm{WL}}$ with respect to $\mathbf{A}$ and equalling the result to zero, resulting in

$$
\begin{aligned}
\mathbf{A} & =\mathbf{C}_{\mathbf{t t}}^{-1} \mathbf{C}_{\mathbf{t s}} \\
& =\left[\begin{array}{cc}
\mathbf{H} \mathbf{H}^{\mathrm{H}}+\sigma_{n}^{2} \mathbf{I}_{N} & \mathbf{H} \mathbf{U H}^{\mathrm{T}} \\
\mathbf{H}^{*} \mathbf{U} \mathbf{H}^{\mathrm{H}} & \mathbf{H}^{*} \mathbf{H}^{\mathrm{T}}+\sigma_{n}^{2} \mathbf{I}_{N}
\end{array}\right]^{-1}\left[\begin{array}{c}
\mathbf{H} \\
\mathbf{H}^{*} \mathbf{U}
\end{array}\right] .
\end{aligned}
$$

Using blockwise matrix inversion, $\mathbf{C}_{\mathbf{t t}}^{-1}$ can be expressed by

$$
\mathrm{C}_{\mathrm{tt}}^{-1}=\left[\begin{array}{ll}
\mathrm{AA} & \mathrm{BB} \\
\mathrm{CC} & \mathrm{DD}
\end{array}\right]
$$

with

$$
\begin{aligned}
\mathbf{A A} & =\left[\sigma_{n}^{2}\left(\mathbf{H}_{\text {mod }}+\sigma_{n}^{2} \mathbf{I}_{N}\right)\right]^{-1}\left(\mathbf{U} \mathbf{H H}^{\mathrm{H}} \mathbf{U}+\sigma_{n}^{2} \mathbf{I}_{N}\right) \\
\mathbf{B B} & =\left[\sigma_{n}^{2}\left(\mathbf{H}_{\text {mod }}+\sigma_{n}^{2} \mathbf{I}_{N}\right)\right]^{-1} \mathbf{H} \mathbf{U} \mathbf{H}^{\mathrm{T}} \\
\mathbf{C C} & =\left[\sigma_{n}^{2}\left(\mathbf{H}_{\text {mod }}+\sigma_{n}^{2} \mathbf{I}_{N}\right)\right]^{-1} \mathbf{H}^{*} \mathbf{U H}^{\mathrm{H}} \\
\mathbf{D D} & =\left[\sigma_{n}^{2}\left(\mathbf{H}_{\bmod }+\sigma_{n}^{2} \mathbf{I}_{N}\right)\right]^{-1}\left(\mathbf{U} \mathbf{H} \mathbf{H}^{\mathrm{H}} \mathbf{U}+\sigma_{n}^{2} \mathbf{I}_{N}\right)
\end{aligned}
$$

and

$$
\mathbf{H}_{\text {mod }}=\mathbf{H H}^{\mathrm{H}}+\mathbf{U} \mathbf{H H}^{\mathrm{H}} \mathbf{U} \text {. }
$$

Analyzing (16), it is possible to see that $\mathbf{H}_{\text {mod }}$ is a diagonal matrix with its diagonal equal to $\left[2\left|H_{1}\right|^{2}, \quad\left(\left|H_{2}\right|^{2}+\right.\right.$ $\left.\left|H_{N}\right|^{2}\right), \quad\left(\left|H_{3}\right|^{2}+\left|H_{N-1}\right|^{2}\right), \ldots 2\left|H_{N / 2+1}\right|^{2}, \ldots\left(\left|H_{3}\right|^{2}+\right.$ $\left.\left.\left|H_{N-1}\right|^{2}\right), \quad\left(\left|H_{2}\right|^{2}+\left|H_{N}\right|^{2}\right)\right]$.

This way, the widely linear equalizer $\mathbf{A}$ can be expressed as

$$
\begin{aligned}
\mathbf{A} & =\mathbf{C}_{\mathbf{t t}}^{-1} \mathbf{C}_{\mathbf{t s}} \\
& =\left[\begin{array}{l}
\mathbf{A}_{1} \\
\mathbf{A}_{2}
\end{array}\right]
\end{aligned}
$$

with the filters $\mathbf{A}_{1}$ (which processes the received signal in the frequency domain) and $\mathbf{A}_{2}$ (which processes its conjugate version) being given by 


$$
\begin{aligned}
\mathbf{A}_{1}= & {\left[\sigma_{n}^{2}\left(\mathbf{H}_{\bmod }+\sigma_{n}^{2} \mathbf{I}_{N}\right)\right]^{-1}\left(\mathbf{U} \mathbf{H} \mathbf{H}^{\mathrm{H}} \mathbf{U}+\sigma_{n}^{2} \mathbf{I}_{N}\right) \mathbf{H}-} \\
& -\left[\sigma_{n}^{2}\left(\mathbf{H}_{\mathrm{mod}}+\sigma_{n}^{2} \mathbf{I}_{N}\right)\right]^{-1} \mathbf{H U H}^{\mathrm{T}} \mathbf{H}^{*} \mathbf{U} \\
= & {\left[\sigma_{n}^{2}\left(\mathbf{H}_{\bmod }+\sigma_{n}^{2} \mathbf{I}_{N}\right)\right]^{-1}\left(\sigma_{n}^{2} \mathbf{H}\right) } \\
= & \left(\mathbf{H}_{\bmod }+\sigma_{n}^{2} \mathbf{I}_{N}\right)^{-1} \mathbf{H}
\end{aligned}
$$

and

$$
\begin{aligned}
\mathbf{A}_{2}= & -\left[\sigma_{n}^{2}\left(\mathbf{H}_{\bmod }++\sigma_{n}^{2} \mathbf{I}_{N}\right)\right]^{-1} \mathbf{H}^{*} \mathbf{U} \mathbf{H}^{\mathrm{H}} \mathbf{H}+ \\
& +\left[\sigma_{n}^{2}\left(\mathbf{H}_{\bmod }+\sigma_{n}^{2} \mathbf{I}_{N}\right)\right]^{-1}\left(\mathbf{U} \mathbf{H H}^{\mathrm{H}} \mathbf{U}+\sigma_{n}^{2} \mathbf{I}_{N}\right) \mathbf{H}^{*} \mathbf{U} \\
= & {\left[\sigma_{n}^{2}\left(\mathbf{H}_{\bmod }+\sigma_{n}^{2} \mathbf{I}_{N}\right)\right]^{-1}\left(\sigma_{n}^{2} \mathbf{H}^{*} \mathbf{U}\right) } \\
= & \left(\mathbf{H}_{\bmod }+\sigma_{n}^{2} \mathbf{I}_{N}\right)^{-1} \mathbf{H}^{*} \mathbf{U} .
\end{aligned}
$$

When transmitting proper signals, $\mathbf{A}$ is reduced to the strictly linear MMSE one, since with proper signals $E\left[\mathbf{s s}^{\mathrm{T}}\right]=0$; thus, taking into account the conjugate version of the received signal in the equalization process does not lead to a performance improvement in this case. This process is very similar to the one done in [14], but better details $\mathbf{A}_{1}$ and $\mathbf{A}_{2}$.

After equalization, an inverse fast Fourier transform (IFFT) is done on $\mathbf{z}$ so that the symbol decision is realized in the time domain, resulting in $\tilde{\mathbf{z}}$ (with size $N$ ). Due to the fact that widely linear processing is employed in the equalizer, the estimated symbols $\tilde{\mathbf{z}}$ at the output of the receiver will be purely real.

\subsection{WL-MMSE DFE equalizer}

When using a WL-MMSE DFE equalizer, the system model is described in Figure 2. This system employs a time-domain feedback filter in addition to a frequencydomain feedforward filter to obtain the symbol estimate.

Assuming that correct past decisions are passed along in the feedback filter, the frequency-domain representation $\mathbf{q}$ of the symbol estimate $\tilde{\mathbf{q}}$ (both with size $N$ ) can be expressed as

$$
\mathbf{q}=\mathbf{B}^{\mathrm{H}} \mathbf{t}-\left(\mathbf{D}-\mathbf{I}_{N}\right) \mathbf{s},
$$

where $\mathbf{B}$ is a $2 N \times N$ matrix corresponding to the feedforward filter and $\mathbf{D}$ is a $N \times N$ matrix with its main diagonal being the $N \times 1$-sized frequency-domain representation of the real-valued time-domain feedback filter $\tilde{\mathbf{d}}=\left[\begin{array}{llll}\tilde{d}_{1} & \tilde{d}_{2} \ldots \tilde{d}_{L_{\tilde{\mathbf{d}}}}\end{array}\right]^{\mathrm{T}}$.

In $\mathbf{q}$, the error (ISI plus noise) component $\mathbf{e}_{\mathrm{WL}-\mathrm{DFE}}$ is given by

$$
\mathbf{e}_{\mathrm{WLLFE}}=\mathbf{B}^{\mathrm{H}} \mathbf{t}-\text { Ds. }
$$

Differentiating the autocorrelation matrix of this error vector with respect to the feedforward filter $\mathbf{B}$ and setting the derivative to zero, we obtain the optimal value of $\mathbf{B}$, expressed as

$$
\begin{aligned}
\mathbf{B} & =\mathbf{C}_{\mathbf{t t}}^{-1} \mathbf{C}_{\mathrm{ts}} \mathbf{D} \\
& =\mathrm{AD} .
\end{aligned}
$$

Replacing (23) in (22) and going to the time domain, we have

$$
\begin{aligned}
\mathbf{e}_{\mathrm{WL}-\mathrm{DFE}}= & \mathbf{D} \mathbf{W}^{-1}\left(\mathbf{A}_{1}^{\mathrm{H}} \mathbf{H}+\mathbf{A}_{2}^{\mathrm{H}} \mathbf{H}^{*} \mathbf{U}\right) \mathbf{W} \mathbf{s}+ \\
& +\mathbf{D W}^{-1} \mathbf{A}_{1}^{\mathrm{H}} \mathbf{W} \mathbf{n}+\mathbf{A}_{2}^{\mathrm{H}} \mathbf{W} \mathbf{n}^{*}-\mathbf{D s} \\
= & \mathbf{D} \mathbf{W}^{-1}\left(\left(\mathbf{H}_{\mathrm{mod}}+\sigma_{n}^{2} \mathbf{I}_{N}\right)^{-1} \mathbf{H}_{\mathrm{mod}}\right) \mathbf{W} \mathbf{s}+ \\
& +\mathbf{D W}^{-1} \mathbf{A}_{1}^{\mathrm{H}} \mathbf{W} \mathbf{n}+\mathbf{A}_{2}^{\mathrm{H}} \mathbf{W} \mathbf{n}^{*}-\mathbf{D s} .
\end{aligned}
$$

Since $\left(\mathbf{H}_{\text {mod }}+\sigma_{n}^{2} \mathbf{I}_{N}\right)^{-1} \mathbf{H}_{\text {mod }}=\mathbf{I}_{N}-\left(\mathbf{H}_{\text {mod }}+\sigma_{n}^{2} \mathbf{I}_{N}\right)^{-1}$ $\sigma_{n}^{2} \mathbf{I}_{N}, \mathbf{e}_{\mathrm{WL}-\mathrm{DFE}}$ can be rewritten as

$$
\begin{aligned}
\mathbf{e}_{\mathrm{WLDFE}}= & -\sigma_{n}^{2} \mathbf{D} \mathbf{W}^{-1}\left(\mathbf{H}_{\mathrm{mod}}+\sigma_{n}^{2} \mathbf{I}_{N}\right)^{-1} \mathbf{W} \mathbf{s}+ \\
& +\mathbf{D} \mathbf{W}^{-1} \mathbf{A}_{1}^{\mathrm{H}} \mathbf{W} \mathbf{n}+\mathbf{A}_{2}^{\mathrm{H}} \mathbf{W} \mathbf{n}^{*} .
\end{aligned}
$$

Thus, the error autocorrelation matrix $C_{\mathbf{e e}}$ can be calculated as

$$
C_{\mathbf{e e}}=\sigma_{n}^{2} \mathbf{D W}^{-1}\left(\mathbf{H}_{\bmod }+\sigma_{n}^{2} \mathbf{I}_{N}\right)^{-1} \mathbf{W D}^{\mathrm{H}} .
$$

Since our goal is to minimize the mean square error (MSE), the trace of the error covariance matrix $C_{\mathbf{e e}}$ should be minimized. This trace is

$$
\operatorname{tr}\left(C_{\mathbf{e e}}\right)=\frac{\sigma_{n}^{2}}{N} \sum_{n=1}^{N} \frac{\left|1-D_{n, n}\right|^{2}}{H_{(\bmod ) n, n}+\sigma_{n}^{2}} .
$$

Using the feedback filter $\tilde{\mathbf{d}}$ in the time domain instead of its frequency domain version $\mathbf{D}$ in (27), we have

$$
\operatorname{tr}\left(C_{\mathbf{e e}}\right)=\frac{\sigma_{n}^{2}}{N} \sum_{n=1}^{N} \frac{\left.\mid 1-\sum_{l=1}^{L_{\tilde{\mathbf{d}}}} \tilde{d}_{l} \exp \left(-j 2 \pi \frac{l n}{N}\right)\right)\left.\right|^{2}}{H_{(\bmod )(n, n)}+\sigma_{n}^{2}} .
$$

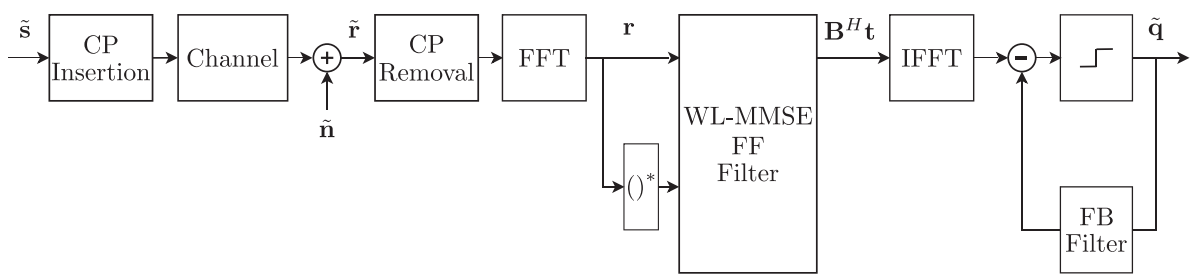

Figure 2 A SC-FDE system employing widely linear MMSE equalization and a decision-feedback equalizer. 
To minimize $\operatorname{tr}\left(C_{\mathbf{e e}}\right)$, we derive (28) with respect to the feedback filter coefficients $\tilde{\mathbf{d}}$ and equal it to zero, obtaining the following linear system:

$$
\mathbf{F} \tilde{\mathbf{d}}=-\mathbf{g} \text {. }
$$

The $L_{\tilde{\mathbf{d}}} \times L_{\tilde{\mathbf{d}}}$ matrix $\mathbf{F}$ and the $L_{\tilde{\mathbf{d}}} \times 1$ column vector $\mathbf{g}$ are expressed, respectively, as

$$
[\mathbf{F}]_{m, l}=\sum_{n=1}^{N} \frac{\exp (-j 2 \pi((n(l-m)) / N))}{H_{(\bmod )(n, n)}+\sigma_{n}^{2}}, 1 \leq m, l \leq L_{\tilde{\mathbf{d}}}
$$

and

$$
[\mathbf{g}]_{m}=\sum_{n=1}^{N} \frac{\exp (j 2 \pi(n m / N))}{H_{(\bmod )(n, n)}+\sigma_{n}^{2}}, 1 \leq m \leq L_{\tilde{\mathbf{d}}}
$$

To initialize the feedback filter, the last $L_{\tilde{\mathbf{d}}}$ symbols of $\tilde{\mathbf{s}}_{\text {CP }}$ can be used. Once $\tilde{\mathbf{d}}$ is determined, B can be calculated by (23). The size of the feedback filter $L_{\tilde{\mathbf{d}}}$ should be equal to the channel length $L_{\tilde{\mathbf{h}}}$ to cancel all the ISI from the previous detected symbols.

\subsection{WL-MMSE Tomlinson-Harashima precoder}

A block diagram for the SC-FDE system using WL-MMSE Tomlinson-Harashima precoding is shown in Figure 3.

In this system model, we consider a single-carrier block transmission, with the block to be transmitted $\tilde{\mathbf{s}}^{\prime}=$ $\left[\tilde{s}_{1}^{\prime} \tilde{s}_{2}^{\prime} \ldots \tilde{s}_{N-L_{\tilde{\mathbf{h}}}^{\prime}}\right]^{\mathrm{T}}$ of size $N-L_{\tilde{\mathbf{h}}}$ composed by symbols belonging to an improper constellation (such as $M$-PAM or $M^{2}$-OQAM) with unit energy. $\tilde{\mathbf{s}}^{\prime}$ then goes to the Tomlinson-Harashima precoder, which consists of a $L_{\tilde{\mathbf{d}}^{-}}$ sized filter $\tilde{\mathbf{d}}^{\prime}=\left[\begin{array}{llll}\tilde{d}_{1}^{\prime} & \tilde{d}_{2}^{\prime} \ldots \tilde{d}_{L_{\tilde{\mathbf{d}}}^{\prime}}^{\prime}\end{array}\right]^{\mathrm{T}}$ and a modulo operator, resulting in $\tilde{\mathbf{x}}^{\prime}$. We recall that the task of the TomlinsonHarashima precoder $\tilde{\mathbf{d}}^{\prime}$ is to use the available channel state information in the transmitter to cancel the interference caused by the channel before transmission. The modulo operator is present to reduce the transmitted signal to a prescribed range, since the precoding operation may increase a given constellation point to an out-of-range value.
The input to the modulo operator in the precoder $\tilde{\mathbf{m}}$ is given by

$$
\tilde{\mathbf{m}}=\tilde{\mathbf{s}}-\sum_{l=1}^{L_{\tilde{\mathbf{d}}}} \tilde{d}^{\prime} \tilde{x}^{\prime \prime}
$$

This modulo operation to $\tilde{\mathbf{m}}$ is done independently on the real and imaginary parts. The output of this modulo operation $\tilde{\mathbf{x}}^{\prime \prime}$ is given by

$$
\begin{aligned}
\tilde{\mathbf{x}}^{\prime \prime} & =\tilde{\mathbf{m}}-2 M\left\lfloor\frac{\operatorname{Re}(\tilde{\mathbf{m}})}{2 M}+\frac{1}{2}\right\rfloor-j 2 M\left\lfloor\frac{\operatorname{Im}(\tilde{\mathbf{m}})}{2 M}+\frac{1}{2}\right\rfloor \\
& =\tilde{\mathbf{m}}+\tilde{\mathbf{a}} .
\end{aligned}
$$

If the real (imaginary) part of $\tilde{\mathbf{m}}$ is greater than $M, 2 M$ is (repeatedly) subtracted from it until the result is less than $M$. If this real (imaginary) part is less than $-M, 2 M$ is (repeatedly) added to it until the result is greater than or equal to $-M$. In other words, $\tilde{\mathbf{m}}$ is reduced modulo $2 M$ to the half-open interval $[-M, M)$, limiting the effective dynamic range of the transmitted signal to this interval. This modulo operation is represented by the sequence $\tilde{\mathbf{a}}$. After this operation, $L_{\tilde{\mathbf{h}}}$ zeros are appended to $\tilde{\mathbf{x}}^{\prime \prime}$ to initialize the state of the precoding filter, resulting in the vector $\tilde{\mathbf{x}}^{\prime}=\left[\begin{array}{llll}\tilde{x}_{1}^{\prime} & \tilde{x}_{2}^{\prime} & \ldots & \tilde{x}_{N}^{\prime}\end{array}\right]^{\mathrm{T}}$ of size $N$. More power is necessary to transmit the precoded symbols when compared to non-precoded ones [15]; however, this penalty becomes negligible with an increase in constellation size.

$\tilde{\mathbf{x}}^{\prime}$ follows the same path of a SC-FDE WL-MMSE-DFE up to the feedback filter (cyclic prefix insertion, channel, cyclic prefix removal, FFT, WL-MMSE equalization, and IFFT). The same modulo operation realized in the transmitter is done in the receiver to $\tilde{\mathbf{y}}^{\prime}$ to map the received data to the interval $(-M, M]$, resulting in the symbol estimate $\hat{\mathbf{s}}^{\prime}$. Only the first $N-L_{\tilde{\mathbf{h}}}$ elements of $\hat{\mathbf{s}}^{\prime}$ are used for the decision.

An equivalent linearized scheme of the system model presented in Figure 3 is shown in Figure 4, following the time-domain THP conversion process made in [16]. In this figure, $\mathbf{K}=\left[\mathbf{H ~ H}^{*}\right]^{\mathrm{T}}$ (with size $N \times 2 N$ ) and $\mathbf{D}^{\prime}$ is a $N \times N$ diagonal matrix with its main diagonal being the $N$-sized Fourier transform of the Tomlinson-Harashima precoder $\tilde{\mathbf{d}}^{\prime}$.

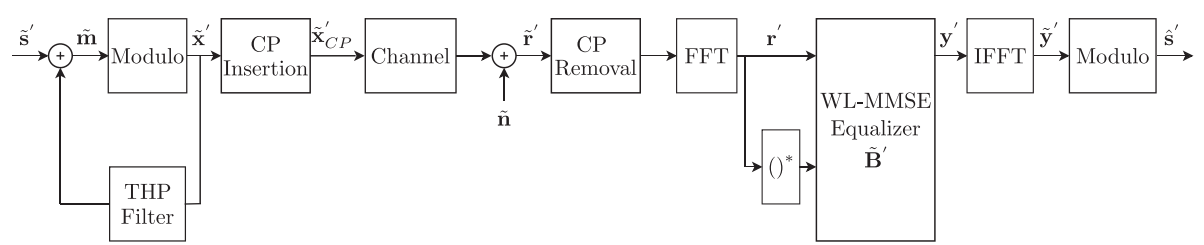

Figure 3 A SC-FDE system employing widely linear MMSE equalization and Tomlinson-Harashima precoding. 


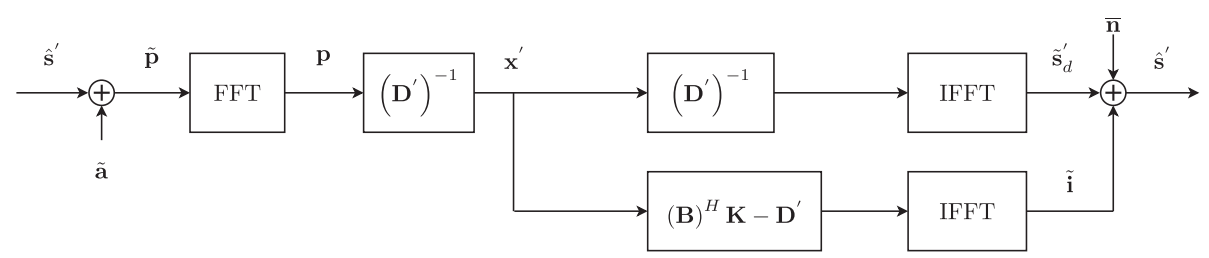

Figure 4 Equivalent system structure for the SC-FDE system employing WL-MMSE equalization and Tomlinson-Harashima precoding.

Figure 4 shows that the symbol estimate $\hat{\mathbf{s}}^{\prime}$ of $\operatorname{size} N-L_{\tilde{\mathbf{h}}}$ is given by

$$
\hat{\mathbf{s}}^{\prime}=\tilde{\mathbf{s}}_{d}^{\prime}+\overline{\mathbf{n}}+\tilde{\mathbf{i}},
$$

where $\tilde{\mathbf{s}}_{d}^{\prime}$ is the desired symbol vector, $\overline{\mathbf{n}}$ the filtered noise, and the remaining interference is expressed by $\tilde{\mathbf{i}}$, all of size $N-L_{\tilde{\mathbf{h}}}$. This way, the error vector $\tilde{\mathbf{e}}^{\prime}$ is

$$
\begin{aligned}
\tilde{\mathbf{e}}^{\prime} & =\overline{\mathbf{n}}+\tilde{\mathbf{i}} \\
& =\mathbf{W}^{-1}\left(\left(\mathbf{B}^{\prime}\right)^{\mathrm{H}} \mathbf{n}\right)+\mathbf{W}^{-1}\left(\left(\mathbf{B}^{\prime}\right)^{\mathrm{H}} \mathbf{K}-\mathbf{D}^{\prime}\right) \mathbf{x}^{\prime} .
\end{aligned}
$$

Using (35), we obtain the mean square error $E^{\prime}$, given by

$$
\begin{aligned}
E^{\prime} & =E\left[|\tilde{\mathbf{e}}|^{2}\right] \\
& =E\left[|\overline{\mathbf{n}}+\tilde{\mathbf{i}}|^{2}\right] .
\end{aligned}
$$

Minimizing (36), we can find that $\mathbf{B}^{\prime}$ and $\tilde{\mathbf{d}}^{\prime}$ are the same as the ones in a SC-FDE system employing a MMSEbased decision-feedback equalizer together with widely linear processing. Thus, the coefficients of the TomlinsonHarashima precoder $\tilde{\mathbf{d}}^{\prime}=\tilde{\mathbf{d}}$ are given by (29), and the widely linear MMSE equalizer $\mathbf{B}^{\prime}=\mathbf{B}$ is given by (23).

\section{Error performance analysis}

\subsection{SINR for the WL-MMSE receiver}

We recall that after the FFT, the received signal and its conjugate version are grouped in the vector $\mathbf{R}$. Both versions are processed together in the frequency domain by the WL-MMSE equalizer $\mathbf{A}$. Thus, the symbol estimate $\tilde{\mathbf{z}}$ is expressed by

$$
\begin{aligned}
\tilde{\mathbf{z}} & =\mathbf{W}^{-\mathbf{1}} \mathbf{A}^{\mathrm{H}} \mathbf{t} \\
& =\mathbf{W}^{-\mathbf{1}} \mathbf{A}^{\mathrm{H}}\left[\begin{array}{c}
\mathbf{H W} \tilde{\mathbf{s}}+\mathbf{n} \\
(\mathbf{H W} \tilde{\mathbf{s}}+\mathbf{n})^{*}
\end{array}\right]
\end{aligned}
$$

is obtained after deprecoding the signal $\mathbf{z}$ at the output of the WL-MMSE equalizer by the IFFT matrix $\mathbf{W}^{-1}$.

We can rewrite (37) in the following way:

$$
\begin{aligned}
\tilde{\mathbf{z}}= & \mathbf{W}^{-\mathbf{1}}\left(\mathbf{A}_{1}^{\mathrm{H}} \mathbf{H}+\mathbf{A}_{2}^{\mathrm{H}} \mathbf{H}^{*} \mathbf{U}\right) \mathbf{W} \tilde{\mathbf{s}}+\mathbf{W}^{-\mathbf{1}} \mathbf{A}_{1}^{\mathrm{H}} \mathbf{n} \\
& +\mathbf{W}^{-\mathbf{1}} \mathbf{A}_{2}^{\mathrm{H}} \mathbf{U n}^{*} .
\end{aligned}
$$

The combined effect of the ISI and the noise in $\tilde{\mathbf{z}}$ is $\mathbf{e}$, given by

$$
\begin{aligned}
\mathbf{e} & =\mathbf{W}^{-\mathbf{1}}\left(\mathbf{A}_{1}^{\mathrm{H}} \mathbf{H}+\mathbf{A}_{2}^{\mathrm{H}} \mathbf{H}^{*} \mathbf{U}-\mathbf{I}_{N}\right) \mathbf{W} \tilde{\mathbf{s}} \\
& +\mathbf{W}^{-\mathbf{1}} \mathbf{A}_{1}^{\mathrm{H}} \mathbf{n}+\mathbf{W}^{-\mathbf{1}} \mathbf{A}_{2}^{\mathrm{H}} \mathbf{U} \mathbf{n}^{*} .
\end{aligned}
$$

With e, we can calculate $E\left[\mathbf{e e}^{\mathrm{H}}\right]$, expressed as

$$
E\left[\mathbf{e e}^{\mathrm{H}}\right]=\mathbf{W}^{-\mathbf{1}}\left(\mathbf{H}_{\bmod }+\sigma_{n}^{2} \mathbf{I}_{N}\right)^{-1} \mathbf{W} .
$$

Since $E\left[\mathbf{e e}^{\mathrm{H}}\right]$ is a circulant matrix, its diagonal elements are all the same. Thus, the MSE for all the elements of $\tilde{\mathbf{z}} \mathrm{MSE}_{\mathrm{WL}}$ is $\frac{1}{N} \operatorname{tr}\left[\mathbf{W}^{-\mathbf{1}}\left(\mathbf{H}_{\bmod }+\sigma_{n}^{2} \mathbf{I}_{N}\right)^{-1} \mathbf{W}\right]$. Note that $\mathrm{MSE}_{\mathrm{WL}}$ is much lower than the MSE given by the strictly linear equalizer, which is $\frac{1}{N} \operatorname{tr}\left[\mathbf{W}^{-1}\left(\mathbf{H}^{\mathrm{H}} \mathbf{H}+\sigma_{n}^{2} \mathbf{I}_{N}\right)^{-1} \mathbf{W}\right]$ [17]. The effective SINR after deprecoding when using a WL-MMSE equalizer $\gamma_{\text {WL-MMSE }}$ is

$$
\gamma_{\mathrm{WL}-\mathrm{MMSE}}=\frac{1}{2}\left(\frac{\gamma N}{\operatorname{tr}\left[E\left[\mathbf{e e}^{\mathrm{H}}\right]\right]}-1\right),
$$

with

$$
\begin{aligned}
\operatorname{tr}\left[E\left[\mathbf{e e}^{\mathrm{H}}\right]\right] & =\frac{1}{2\left|H_{1}\right|^{2}+\sigma_{n}^{2}}+\frac{1}{2\left|H_{N / 2+1}\right|^{2}+\sigma_{n}^{2}}+ \\
& +\sum_{i=2}^{N / 2} \frac{2}{\left|H_{i}\right|^{2}+\left|H_{N+2-i}\right|^{2}+\sigma_{n}^{2}}
\end{aligned}
$$

and $\gamma=E_{s} / \sigma_{n}^{2}$. The division by 2 in (41) is because the final symbol decision is only done on the real estimate [18]. Since [14] does not specify well $\mathbf{A}_{1}$ and $\mathbf{A}_{2}$, (41) clarifies the calculation of the SINR for a SC-FDE system using widely linear MMSE equalization in the SISO case.

\subsection{SINR for the WL-MMSE DFE receiver}

For the SC-FDE system using WL-MMSE DFE equalization, its MSE can be expressed (using the method described in [19]) as

$$
\mathrm{MSE}_{\mathrm{WL}-\mathrm{DFE}}=\exp \left(\frac{1}{N} \sum_{n=1}^{N} \log \left(\frac{1}{1+\gamma H_{(\bmod )(n, n)}}\right)\right) .
$$

The mean square error expressed in (43) does not take into account the error propagation effect that can be caused by erroneous previous decisions. This MSE is 
again lower than the one obtained by the strictly linear solution, given by [20]

$$
\mathrm{MSE}_{\mathrm{DFE}}=\exp \left(\frac{1}{N} \sum_{n=1}^{N} \log \left(\frac{1}{1+\gamma\left|H_{n}\right|^{2}}\right)\right) .
$$

The SINR for the SC-FDE system using WL-MMSE DFE equalization is given by

$$
\gamma_{\mathrm{WL}-\mathrm{DFE}}=\frac{1}{2}\left(\frac{1}{\mathrm{MSE}_{\mathrm{WL}-\mathrm{DFE}}}-1\right)
$$

Again, we divide by 2 to obtain the effective SINR for the system using widely linear equalization.

\subsection{SINR for the WL-MMSE-THP precoder}

The MSE for the WL-MMSE-THP SC-FDE system can be expressed as

$$
\mathrm{MSE}_{\mathrm{WL}-\mathrm{THP}}=\exp \left(\frac{1}{N} \sum_{n=1}^{N} \log \left(\frac{1}{1+\frac{\gamma}{\eta} H_{(\bmod )(n, n)}}\right)\right),
$$

with $\eta=\frac{M^{2}}{M^{2}-1}$. Let us note that this MSE is the same one from a WL-MMSE DFE one outside of a precoding loss factor $\eta$ dependent on the constellation size [15].

Finally, to calculate the error probability $P_{\mathrm{e}}$ conditional to a channel realization in each of the previous cases, the following equation is used:

$$
P_{\mathrm{e}}=\alpha Q(\sqrt{\beta \gamma}),
$$

where $\alpha$ and $\beta$ are constellation-specific parameters [21]; $\gamma$ can be $\gamma_{\text {WL-MMSE}}, \gamma_{\text {WL-DFE }}$, or $\gamma_{\text {WL-THP }}$; and $Q(x)=$ $\frac{1}{\sqrt{2 \pi}} \int_{x}^{\infty} \exp \frac{-t^{2}}{2} d t$. The unconditional error probability is obtained by averaging over all the conditional error probabilities corresponding to the channel realizations.

\section{Simulation results}

Simulation results are presented in this section to validate the use of widely linear MMSE-based equalization and precoding to obtain an error performance improvement for different block sizes and channel models. For the simulations presented in this section, the cyclic prefix size used is the minimum sufficient to eliminate the interblock interference, and the power loss caused by the redundancy introduced by the cyclic prefix is taken into account in the SNR calculation. A sampling frequency of $10 \mathrm{MHz}$ was used. To calculate the final bit error performance in the Monte Carlo simulations, a minimum of 400 errors were taken into account for each point; for the method presented in this paper, 5,000 independent channel realizations were used to obtain the unconditional error probability. Channel estimation in the receiver is assumed to be perfect (unless noted otherwise), channel fading is considered to be quasistatic (time-invariant during each transmitted block), and other system imperfections are not taken into account. For THP systems, the precoder size $L_{\tilde{\mathbf{d}}}$ is equal to the channel length $L_{\tilde{\mathbf{h}}}$. In simulations using channel coding, a mother convolutional code with $R=1 / 2,(171,133)_{8}$ code followed by a block interleaver is used at the transmitter; in the receiver, a block de-interleaver followed by a soft-decision Viterbi decoder is used. Higher code rates are obtained through puncturing.

Figure 5 shows the uncoded Monte Carlo results for a SC-FDE system using WL-MMSE equalization compared to the results provided by (47) for transmission symbols drawn from a BPSK constellation $(\alpha=\beta=1)$, a block size $N=128$ and the ITU-T Vehicular A channel model. For the systems employing a time-domain DFE, its length $L_{\tilde{\mathbf{d}}}$ is equal to the channel length $L_{\tilde{\mathbf{h}}}$. For reference, the error performance of SC-FDE systems using strictly linear MMSE equalization is also shown. It is possible to see that the use of the analysis presented in this paper gives consistent results when compared to the Monte Carlo simulation results throughout the $E_{b} / N_{0}$ range. The utilization of the WL-MMSE equalizers brings a performance gain when compared to the strictly linear MMSE ones in the entire $E_{b} / N_{0}$ range, due to the complete use of the second-order statistics made available by the improper signal. For reference, the error performance results of SCFDE systems using linear iterative IB-DFE equalization [22] (with four iterations) are also presented. It can be seen that the error performance obtained by the WL-DFE system is close to the one obtained by iterative equalization, but without the added decision delay caused by the

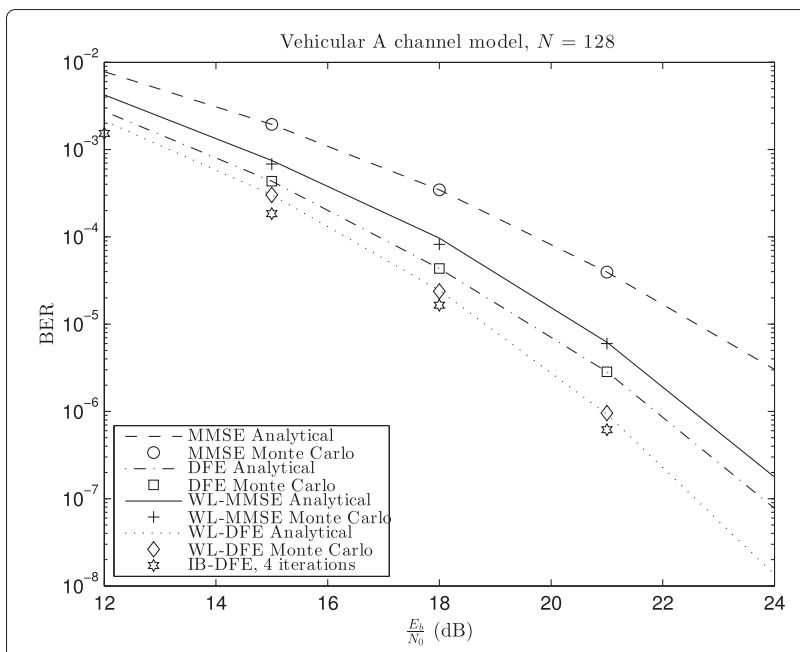

Figure 5 Error performance for SC-FDE systems with $N=128$ and BPSK modulation. 
iterative process. Results using a quadrature phase-shift keying (QPSK) constellation for the strictly linear receiver and an offset QPSK (OQPSK) constellation for the widely linear one will be the same as the ones presented in this figure.

Figure 6 presents uncoded results for $N=512$, the ITU-T Pedestrian B channel model and again a BPSK constellation. The same conclusions made for the previous case can also be stated for this scenario. For high $E_{b} / N_{0}$ ratios, the Monte Carlo simulation can be very timeconsuming for the WL-MMSE receiver, due to low BER values. Thus, the use of the analytical method presented in this paper allows us to derive the system performance in less time.

Results for the same scenario employed in Figure 5, but with 16-QAM/OQAM constellations in Figure 7 and 64-QAM/OQAM constellations in Figure 8, are shown. It is possible to see that the performance advantage between the widely linear equalizer and the strictly linear one in the case where a time-domain DFE is not used increases when the constellation size grows. This can be explained by the fact that the WL-MMSE feedforward filter is more effective in eliminating the ISI when compared to the strictly linear MMSE feedforward filter. With a time-domain DFE, the advantage for the widely linear equalizer remains the same with the increase of the constellation size because the feedback filter cancels some of the residual ISI; thus, some of the performance advantage seen in the previous case is negated. It is interesting to note that the system with a WL-MMSE DFE transmitting symbols from a 64-OQAM constellation has better error performance that the system transmitting symbols from a 16-QAM constellation using regular linear MMSE equalization.

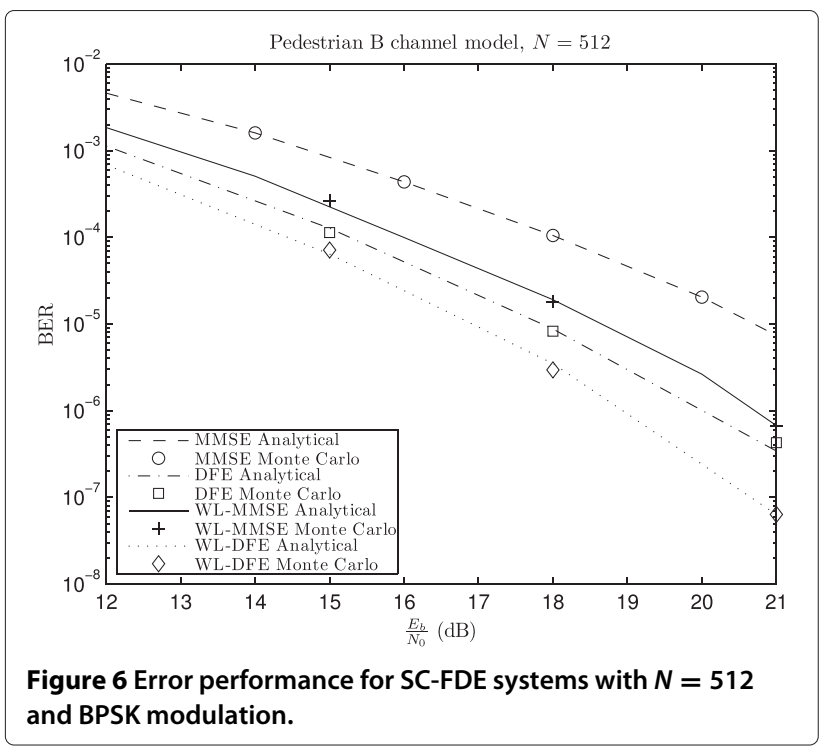

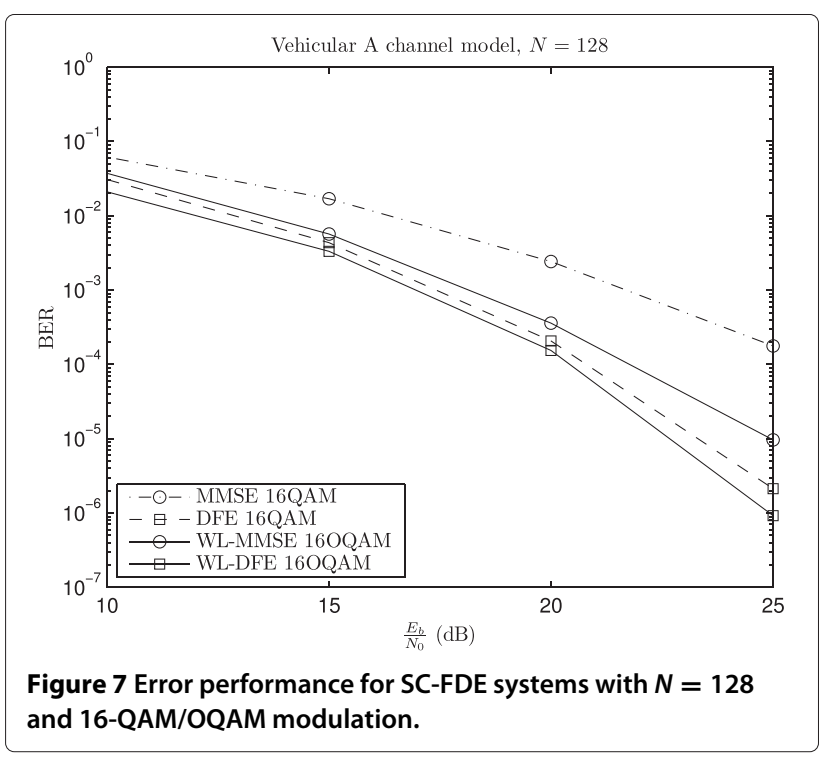

In Figure 9, results for $N=128$, a OQPSK constellation, the Vehicular A channel model, but this time using convolutional coding, are presented. In this scenario, the performance gain from using the WL-MMSE equalizer is also observed, with its advantage growing with a weaker code ratio. This happens because the stronger code ratios help compensate some of the performance advantage which comes with the usage of the widely linear equalizer. For systems using a feedback filter, coding is not directly applicable due to the effect of error propagation in the feedback filter, which causes a significant amount of burst errors in the Viterbi decoder [4]. Efforts to overcome this problem in decision-feedback equalizers have been discussed in $[23,24]$.

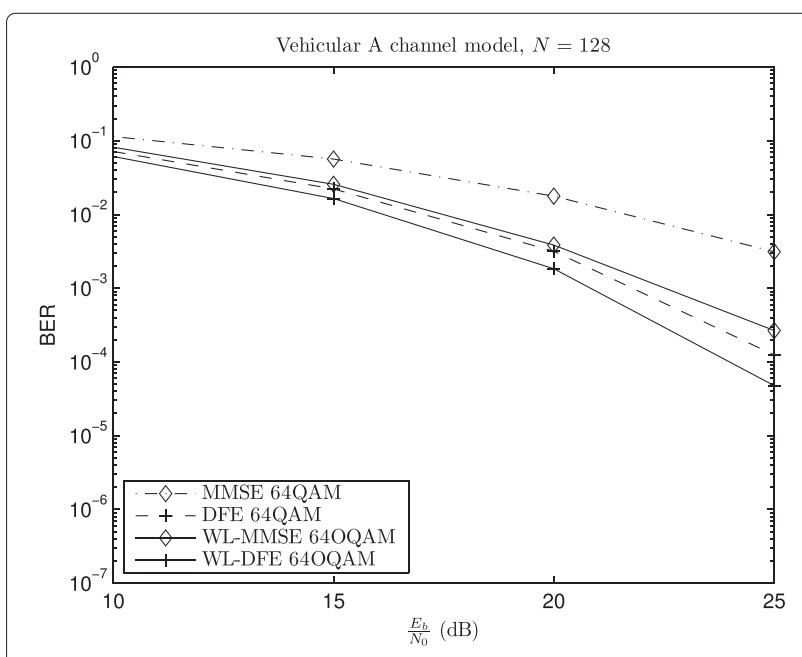

Figure 8 Error performance for SC-FDE systems with $N=128$ and 64-QAM/OQAM modulation. 


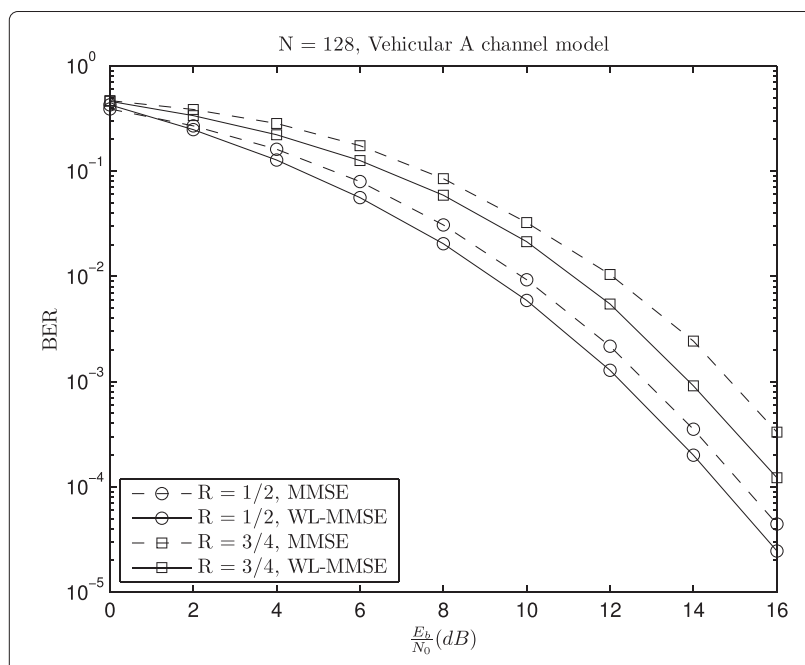

Figure 9 Error performance for SC-FDE systems with $N=128$ and BPSK modulation using convolutional coding.

In the simulations made to obtain the previous results using decision-feedback equalizers, the length of the feedback filter $L_{\tilde{\mathbf{d}}}$ was always the length of the channel's impulse response $L_{\tilde{\mathbf{h}}}$. Figure 10 shows the effect of changing this feedback filter length $L_{\tilde{\mathbf{d}}}$ on the error performance of SC-FDE DFE systems. Feedback filter sizes of $L_{\tilde{\mathbf{h}}} / 2$, $L_{\tilde{\mathbf{h}}} / 4$, and $L_{\tilde{\mathbf{h}}} / 8$ were considered. The system using widely linear equalization has its error performance less sensitive to the error propagation effect caused by the smaller feedback filters when compared to the system using strictly linear equalization due to the increased effectiveness of the feedforward filter in this case. With smaller feedback filters, the computational complexity needed to calculate their coefficients in (30) and (31) is reduced.

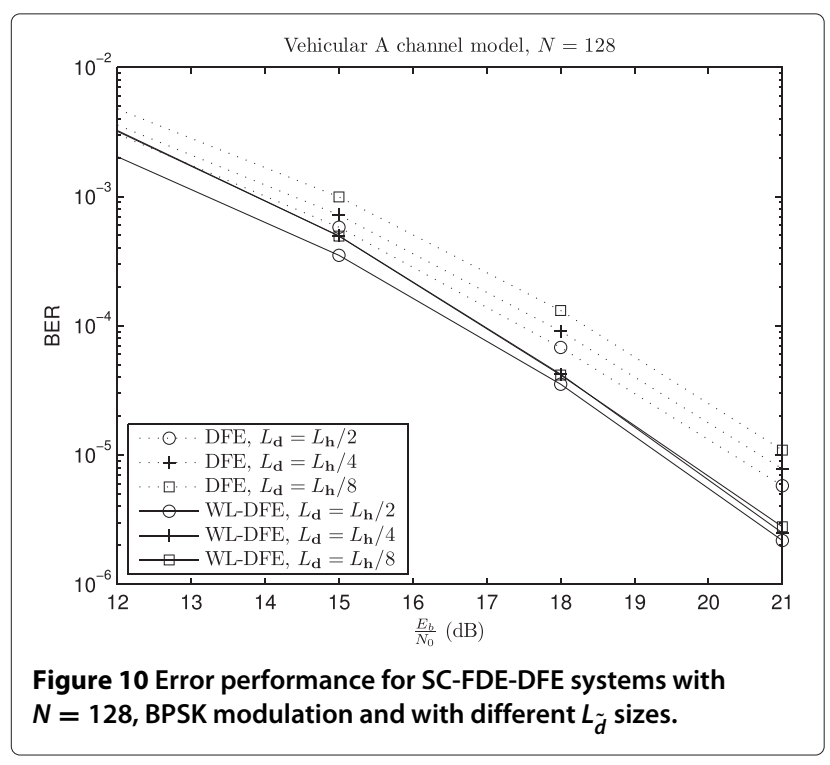

The error performance results of widely linear MMSE Tomlinson-Harashima precoding applied to a SC-FDE system compared to its strictly linear version for a BPSK constellation, $N=128$ and the ITU-T Vehicular A channel model, are shown in Figure 11. In these simulations, the channel estimation in the receiver and channel state information in the transmitter are assumed to be perfect. It is possible to see that the system using widely linear processing outperforms its strictly linear counterpart. This is again due to the complete use of the secondorder statistics made available by the transmitted signal. As stated before, this error performance is the same as the one obtained by the widely linear systems employing a decision-feedback equalizer outside of a precoding loss factor. Since this precoding loss is smaller in higher-order constellations, the error performance results of SC-FDE systems using widely linear MMSE Tomlinson-Harashima precoding when using 16-OQAM and 64-OQAM constellations will be almost identical to the ones from WL-DFE systems presented in Figures 7 and 8.

As said before, the usage of channel coding in systems using Tomlinson-Harashima precoding is possible, unlike in systems employing non-modified decision-feedback equalizers. The results for Tomlinson-Harashima precoded systems using convolutional coding are presented in Figure 12. In this scenario, the performance gain from using widely linear-based precoding is also observed, with its advantage growing with a weaker code ratio.

The previous results when using Tomlinson-Harashima precoding assumed perfect channel estimation in the receiver and perfect channel state information at the transmitter; however, this is an unlikely scenario in real conditions because of channel variations. As stated before, Tomlinson-Harashima precoded systems rely on

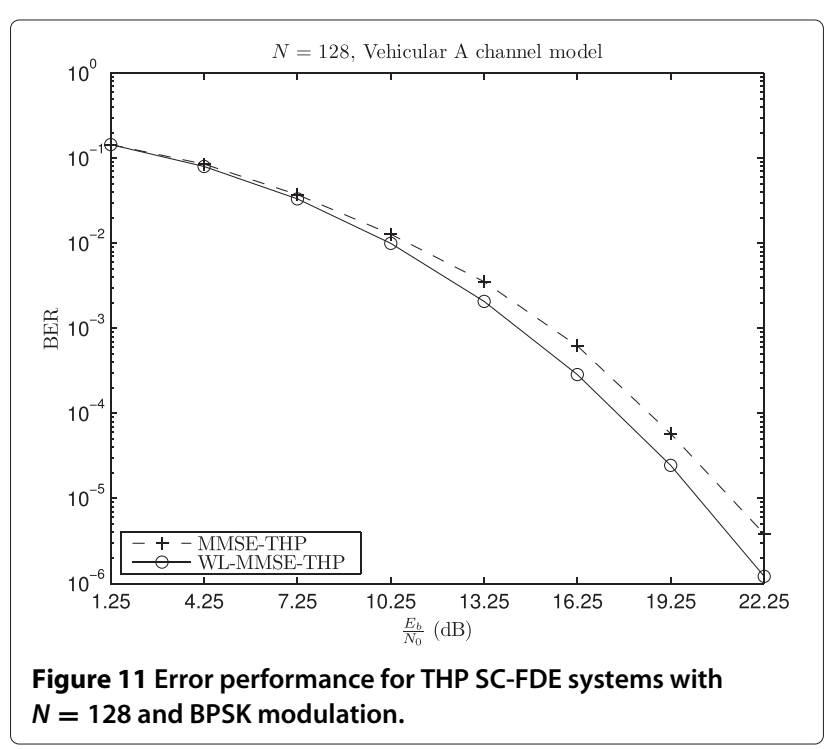




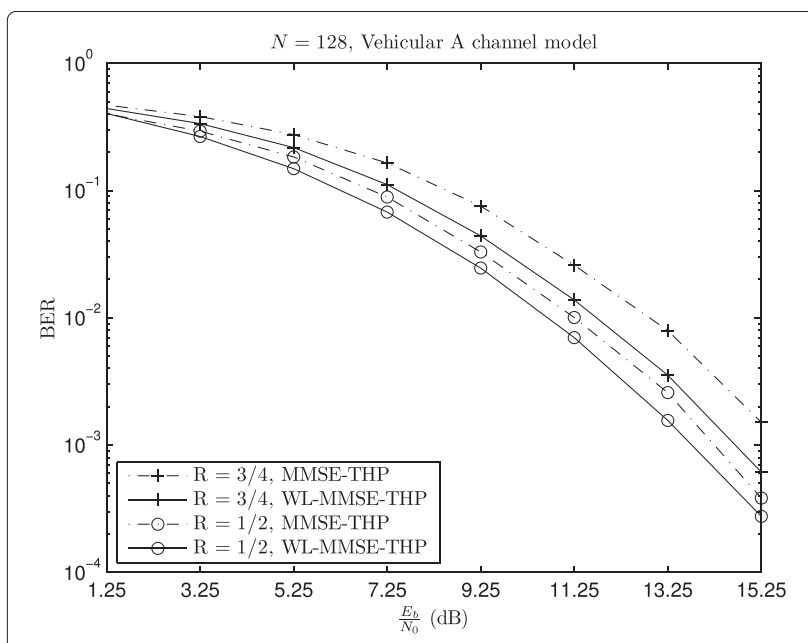

Figure 12 Error performance for SC-FDE-THP systems with $N=128$ and BPSK modulation using convolutional coding.

complete channel state information in the transmitter, which in turn needs perfect channel estimation in the receiver. A comparison on the impact of channel estimation errors and imperfect CSI in the error performance of MMSE-THP SC-FDE systems is presented in Figure 13 for $\frac{E_{b}}{N_{0}}=19.25 \mathrm{~dB}, N=128$, and the Vehicular A channel model. The imperfect channel estimates can be expressed as $\mathbf{H}_{\mathrm{e}}=\mathbf{H}+\mathbf{E}_{\mathbf{H}}$, where $\mathbf{E}_{\mathbf{H}}$ is the channel estimation error matrix, with its diagonal composed of zero-mean Gaussian distributed random variables with variance $\sigma_{\mathrm{e}}^{2}$ [25]. This imperfect channel estimate is then passed to the transmitter, which will have erroneous CSI. Even with an increase of the error variance $\sigma_{\mathrm{e}}^{2}$, the SC-FDEsystem using widely linear processing outperforms its strictly linear counterpart.

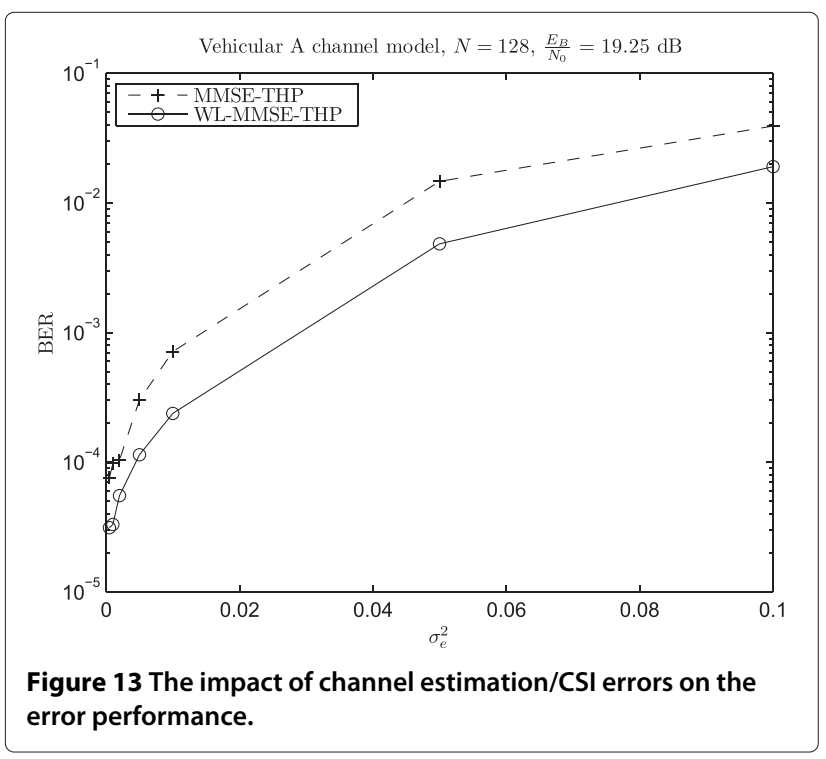

\section{Conclusions}

This paper presented novel SC-FDE systems using widely linear MMSE-based equalization, decision-feedback equalization, and Tomlinson-Harashima precoding. The use of widely linear processing brings, when the transmitter uses improper constellations, a performance gain compared to when common strictly linear MMSE processing is used. With respect to SC-FDE systems using MMSE-DFE equalization, together with the performance gain, the use of widely linear processing also makes the error performance less sensitive to the feedback filter size. The error performance gain of Tomlinson-Harashima precoded systems using widely linear processing is also observed when there are channel estimation/CSI errors. Possibilities for future work include combining widely linear processing with the pragmatic frequency-domain equalization method for SC-FDE systems employing offset modulation and pulse shaping [26,27].

\section{Competing interests}

The authors declare that they have no competing interests.

\section{Acknowledgements}

This work has been partially sponsored by National Council for Scientific and Technological Development (CNPq) and CAPES/COFECUB project (544/07).

\section{Author details}

${ }^{1}$ Electronics Department, Federal University of Technology - Paraná, Curitiba 80230-901, Brazil. ${ }^{2}$ Communications Research Group, Federal University of Santa Catarina, Florianópolis 88040-900, Brazil. ${ }^{3}$ CEDRIC/LAETITIA Laboratory, Conservatoire National des Arts et Métiers, 75141 Paris Cédex 03, France.

Received: 7 October 2013 Accepted: 12 July 2014

Published: 7 August 2014

\section{References}

1. H Sari, G Karam, I Jeanclaude, Transmission techniques for digital terrestrial TV broadcasting. IEEE Commun. Mag. 33(2), 100-109 (1995)

2. D Falconer, SL Ariyavisitakul, A Benyamin-Seeyar, B Eidson, Frequency domain equalization for single-carrier broadband wireless systems. IEEE Commun. Mag. 40(4), 58-66 (2002)

3. N Benvenuto, STomasin, On the comparison between OFDM and single carrier modulation with a DFE using a frequency-domain feedforward filter. IEEE Trans. Commun. 50(6), 947-955 (2002)

4. G Huang, A Nix, S Armour, Decision feedback equalization in SC-FDMA, in IEEE 19th International Symposium on Personal, Indoor and Mobile Radio Communications (Cannes, 15-18 Sept. 2008)

5. M Tomlinson, New automatic equaliser employing modulo arithmetic. Electron. Lett. 7, 138-139 (1971)

6. H Harashima, H Miyakawa, Matched-transmission technique for channels with intersymbol interference. IEEE Trans. Comm. 20(4), 774-780 (1972)

7. $Y$ Zhu, K Letaief, Frequency domain pre-equalization with transmit precoding for MIMO broadcast wireless channels. IEEE J. Sel. Area. Comm. 26(2), 389-400 (2008)

8. M Noune, A Nix, Frequency-domain precoding for single carrier frequency-division multiple access. IEEE Comm. Mag. 47(6), 68-74 (2009)

9. B Picinbono, On circularity. IEEE Trans. Signal Process. 42(12), 3473-3482 (1994)

10. B Picinbono, P Chevalier, Widely linear estimation with complex data. IEEE Trans. Signal Process. 43(8), 2030-2033 (1995)

11. PJ Schreier, LL Scharf, CT Mullis, Detection and estimation of improper complex random signals. IEEE Trans. Inform. Theor. 51(1), 306-312 (2005)

12. P Chevalier, F Pipon, New insights into optimal widely linear array receivers for the demodulation of BPSK, MSK, and GMSK signals corrupted by noncircular interferences - application to SAIC. IEEE Trans. Signal Process. 54(3), 870-883 (2006) 
13. D Darsena, G Gelli, F Verde, Universal linear precoding for NBI-proof widely linear equalization in MC systems. EURASIP J. Wireless Commun. Netw. 2008, 1-14 (2008)

14. Z Lin, P Xiao, B Vucetic, M Sellathurai, Analysis of receiver algorithms for LTE SC-FDMA based uplink MIMO systems. IEEE Trans. Wireless Comm. 9(1), 60-65 (2010)

15. RFH Fischer, Precoding and Signal Shaping for Digital Transmission. (Wiley, New York, 2002)

16. RD Wesel, JM Cioffi, Achievable rates for Tomlinson-Harashima precoding. IEEE Trans. Inform. Theor. 53(1), 824-831 (1998)

17. A Tajer, A Nosratinia, Diversity order in ISI channels with single-carrier frequency-domain equalizers. IEEE Trans. Wireless Comm. 9(3), 1022-1032 (2010)

18. A Mirbagheri, KN Plataniotis, S Pasupathy, An enhanced widely linear CDMA receiver with OQPSK modulation. IEEE Trans. Commun. 54(2), 261-272 (2006)

19. J Salz, Optimum mean-square decision feedback equalization. Bell Syst. Tech. J. 52, 1341-1373 (1973)

20. C Panazio, A de Paula, An uncoded BER comparison between DFE-SCCP and OFDM using a convex analysis framework, in IEEE International Symposium on Circuits and Systems - IEEE ISCAS 2011 (Rio de Janeiro, 15-18 May, 2011)

21. JG Proakis, Digital Communications, 4th edn. (McGraw-Hill, Upper Saddle River, 2000)

22. N Benvenuto, S Tomasin, Iterative design and detection of a DFE in the frequency domain. IEEE Trans. Commun. 53(11), 1867-1875 (2005)

23. MV Eyuboglu, Detection of coded modulation signals on linear, severely distorted channels using decision-feedback noise prediction with interleaving. IEEE Trans. Commun. 36(4), 401-409 (1988)

24. $Y Z$ Zhu, KB Letaief, Single carrier frequency domain equalization with noise prediction for broadband wireless systems. GLOBECOM. 5, 3098-3102 (2004). Dallas, 29 Nov.-3 Dec. 2004

25. J Maurer, J Jalden, G Matz, Multi-threshold TOP_full-diversity vector perturbation precoding with finite-rate feedforward, in Proceedings of the Asilomar Conference on Signals, Systems and Computers (Pacific Grove, 26-29 Oct. 2008), pp. 428-432

26. M Luzio, R Dinis, P Montezuma, SC-FDE for offset modulations: an efficient transmission technique for broadband wireless systems. IEEE Trans. Commun. 60(7), 1851-1861 (2012)

27. M Luzio, R Dinis, P Montezuma, Pragmatic frequency domain equalization for single carrier with offset modulations. IEEE Trans. Wireless Commun. 12(9), 4496-4505 (2013)

\section{Submit your manuscript to a SpringerOpen ${ }^{\circ}$ journal and benefit from:}

- Convenient online submission

- Rigorous peer review

- Immediate publication on acceptance

- Open access: articles freely available online

- High visibility within the field

- Retaining the copyright to your article 